\title{
Early oncological outcomes and accuracy of risk stratification and tailoring surgical staging based on preoperative histology, Ca125 and MRI in endometrial cancer: a prospective cohort study
}

\author{
Jency Mathews*, Seethal Abraham
}

Department of Surgical Oncology, Pushpagiri Medical College, Tiruvalla, Kerala, India

Received: 27 August 2019

Accepted: 30 September 2019

\author{
*Correspondence: \\ Dr. Jency Mathews, \\ E-mail: jencymathews@pushpagiri.in
}

Copyright: ( $)$ the author(s), publisher and licensee Medip Academy. This is an open-access article distributed under the terms of the Creative Commons Attribution Non-Commercial License, which permits unrestricted non-commercial use, distribution, and reproduction in any medium, provided the original work is properly cited.

\begin{abstract}
Background: momentum to select patients who will benefit from the extensive procedures. However, the parameters used for risk stratification have variable accuracy outside of tertiary cancer centres. This study looks into the accuracy of risk stratification using preoperative histology, MRI and $\mathrm{Ca} 125$ levels and the oncological outcomes after tailoring surgical staging based on the risk stratification by combining the three variables in a suburban centre with a growing cancer population.

Methods: This prospective observational cohort study was undertaken in a suburban cancer center in Pushpagiri Medical College, Tiruvalla, Kerala between June 2014 and December 2018. All patients underwent surgical staging with hysterectomy and salpingo oophorectomy as the least procedure. Lymphadenectomy was tailored according to the preoperative risk grouping and changed only in the presence of gross findings at surgery. Adjuvant treatment and follow up data obtained and collected in Microsoft Excel and analysed using statistical software SPSS version 22.

Results: Of 47 patients recruited for the study, 35 patients were available for final analysis. Preoperative histology was accurate in $73 \%$. There was $20 \%$ overestimation and $8 \%$ underestimation. Ca 125 levels were elevated in $15 \%$. MRI had an overall sensitivity of $74 \%$ and specificity of $60 \%$. When MRI, Ca125 and histology were combined together, there was patients were deemed to be high risk. On final risk grouping, 9 patients were down staged and none were upstaged.

Conclusions: Preoperative histology, MRI and ca 125 levels have moderate accuracy individually as preoperative risk determinates. The three parameters combined together show high specificity and PPV for preoperative risk stratification and the risk stratification has not been detrimental with respect to oncological outcomes of recurrence.
\end{abstract}

Keywords: Endometrial cancer, Histology, MRI and Ca-125, Outcomes, Preoperative Risk Stratification

\section{INTRODUCTION}

Uterine cancer shows an increasing trend in India among both urban and rural populations. It is already the most common gynaecological malignancy in developed countries. ${ }^{1}$ Surgical staging of uterine cancers is the gold standard unchanged by advances in imaging or adjuvant treatment. The standard surgical staging procedure in the non-metastatic setting includes a peritoneal cytology, total hysterectomy with bilateral salpingo-oophorectomy, pelvic and para-aortic node dissections and omentectomy in addition any tumor deposits excision. ${ }^{2}$

But the extent of procedures required for adequate staging of uterine cancers has always been debated. Early stage endometrial cancer has a good prognosis and 
treatment morbidity has long term consequences. The presence of co-morbidities like obesity and advanced age places the need to modify the extent of surgical staging in apparent early stage disease. Foregoing a complete lymphadenectomy procedure can significantly contribute to saving valuable operating theatre time. The increasing cancer burden also places the need to use resources judiciously. Several preoperative prediction models and preoperative risk grouping methods have been studied to tailor the extent of staging procedure with varying success but none have been formally incorporated into the guidelines. ${ }^{2}$

Such preoperative risk based staging procedures is gaining popularity in many centres with preoperative histology, ca-125 and magnetic resonance imaging guiding the extent of surgical staging. ${ }^{3,4}$ This prospective cohort study is an attempt to evaluate the oncological outcomes of the preoperative risk stratification and the accuracy of each of these preoperative parameters, the efficacy of combining the 3 parameters for preoperative staging, tailoring the surgical staging accordingly and its early oncological outcomes.

\section{METHODS}

All consecutive patients who underwent surgical staging for suspected or proven uterine cancers between June 2014 and December 2018 at Pushpagiri Hospital Cancer Centre, Tiruvalla, Kerala, India were included in the study. The exclusion criteria were non-malignant diseases of the uterus and uterine sarcomas on final pathology report. All patients had a preoperative histology assessment, CA-125 levels and preoperative imaging with MRI pelvis. All patients underwent comprehensive surgical staging. Adjuvant treatment was planned according to tumor board. The research protocol was approved by the institutional ethics board.
Demographic details (age, BMI, menopausal status, parity status) and disease related details (preoperative histology, radiology including ultrasonography and MRI, Ca-125, co-morbidities, surgical approach, extent of staging procedures, final histopathological details, postoperative follow up outcomes) were prospectively entered into a Microsoft Excel database. Statistical analysis was done using SPSS version 22 software. The sensitivity, specificity, positive predictive value (PPV), negative predictive value (NPV), likelihood ratio (LR+, LR-) of the preoperative assessment were calculated for each endpoint, together with $95 \%$ confidence intervals $(95 \% \mathrm{CI})$. The percentage of underestimation or overestimation and accuracy rate were also calculated.

\section{Definition of risk groups and extent of surgical staging}

Patients were grouped into 3 risk groups based on preoperative risk assessment based on preoperative histology, MRI pelvis and Ca125 levels as detailed in Table 1. Laparoscopy, laparotomy or hybrid procedure (laparoscopic assisted) was performed at the discretion of the operating surgeon. Peritoneal lavage was taken in all patients. Extra-fascial Hysterectomy with bilateral salpingo oophorectomy was the least procedure done. Omentectomy or omental sampling was done based on preoperative histology (sampling for low risk histology and resection for high risk histology) and suspicious intraoperative findings. Lymphadenectomy was done according to the preoperative risk grouping and modified based on intraoperative findings as follows:

Low risk: No lymphadenectomy or lymph node sampling only.

High risk: Comprehensive pelvic lymph node dissection.

Table 1: Preoperative risk grouping.

\begin{tabular}{|llll|}
\hline Risk grouping & Preoperative risk grouping & Ca -125 & MRI \\
\hline Low & Grade 1 endometrioid ca & $<35$ IU & $<50 \%$ myometrial invasion, 'no nodal metastases \\
\hline High & $\begin{array}{l}\text { Grade 2, 3 endometrioid ca } \\
\text { Non endometrioid histology }\end{array}$ & $>35$ IU & $\begin{array}{l}>50 \% \text { myometrial invasion, any nodal metastases } \\
\text { Any extra uterine spread }\end{array}$ \\
\hline
\end{tabular}

Table 2: New risk grouping by ESMO-ESGO-ESTRO consensus.

\begin{tabular}{|c|c|}
\hline Risk group & Risk factors \\
\hline Low & Stage I endometrioid, grade $1-2,<50 \%$ myometrial invasion, LVSI negative \\
\hline Intermediate & Stage I endometrioid, grade $1-2, \geq 50 \%$ myometrial invasion, LVSI negative \\
\hline High-intermediate & $\begin{array}{l}\text { Stage I endometrioid, grade } 3,<50 \% \text { myometrial invasion, regardless of LVSI status } \\
\text { Stage I endometrioid, grade } 1-2, \text { LVSI unequivocally positive, regardless of depth of invasion }\end{array}$ \\
\hline High & Stage I endometrioid, grade $3, \geq 50 \%$ myometrial invasion, regardless of LVSI status \\
\hline Advanced-metastastic & $\begin{array}{l}\text { Stage II, stage III endometrioid, no residual disease, non-endometrioid (serous or clear-cell or } \\
\text { undifferentiated carcinoma or carcinosarcoma) }\end{array}$ \\
\hline
\end{tabular}


Para aortic node sampling or node dissection was performed in all grade 3 or aggressive histology or if preoperative MRI was suspicious of para aortic nodal metastases or if intraoperatively the pelvic lymph nodes were suspicious for metastases.

Postoperatively, based on the age, final histology, nodal metastases and presence of LVSI patients were risk grouped according to the ESMO-ESGO-ESTRO guidelines (Table 2) and adjuvant treatment was planned after tumor board. Patients were followed up till $30^{\text {th }}$ April 2019 and the follow up data was collected.

\section{RESULTS}

The 47 consecutive patients underwent primary surgical staging during the study period. 12 patients were excluded from the study as final histology showed sarcoma variants of uterine cancers, carcinosarcoma and non-malignant diseases of uterus. 35 patients were available for the final analysis.

The clinical and pathological characteristics of the patients are detailed in Table 3. The median age of patients with endometrial cancers was 56 years, range (42-80). $31(89 \%)$ patients had Type 1 endometrial cancer and $4(11 \%)$ patients had Type 2 endometrial cancers. 2 patients had synchronous cancers, one locally advanced rectal cancer and one synchronous ovarian cancer along with endometrial cancer. One patient had developed endometrial carcinoma as the metachronous cancer after recurrent colon cancer.

Preoperative histology was accurate in $73 \%$. There was $20 \%$ overestimation and $8 \%$ underestimation with respect to type and grade. In 1 patient, the tumor type changed from type 2 tumor to type 1 tumor on final histology and vice versa in 1 patient. 2 patients had no residual malignancy in the final histology. 2 patients had grade of the tumor changed from grade 1 on preoperative histology to grade 2 on final histology. Ca -125 levels was elevated $(>35 \mathrm{IU})$ in $15 \%$ patients. All these patients with elevated $\mathrm{Ca}-125$ levels had an MRI stage of $1 \mathrm{~b}$ or higher except in 1 patient who had stage 1a endometrioid carcinoma. This patient with $\mathrm{Ca}-125$ levels $>1000$ had unexplained ascites at 4 months after surgery and succumbed to cachexia although all subsequent imaging was negative for metastases.

The results of the MRI in detecting myometrial invasion, cervical invasion and lymph node invasion are listed in Table 4. MRI had an overall sensitivity of $74 \%$ and specificity of $60 \%$. Stage wise accuracy of MR imaging and concordance with final histology is shown in Table 5. Myometrial invasion was overestimated in $20 \%$ and underestimated in $17 \%$ with an accuracy of $63 \%$. Cervical invasion was accurate in $83 \%$, with overestimation in $6 \%$ and underestimated in $11 \%$. Nodal invasion on MRI was accurate in $81 \%$ with $16 \%$ overestimation and $3 \%$ underestimation. The overestimation was seen in pelvic lymph nodes only and the underestimation was in para aortic nodes.

Table 3: Patient characteristics $(n=35)$.

\begin{tabular}{|c|c|}
\hline Sr. no. & Patient details \\
\hline \multirow{3}{*}{1} & Age-median 56 years (range 42-80) \\
\hline & Age $<60$ years $-15(49 \%)$ \\
\hline & $>60$ years $-20(57 \%)$ \\
\hline \multirow{3}{*}{2} & Menopausal status \\
\hline & Premenopausal - $9(26 \%)$ \\
\hline & Postmenopausal - $26(74 \%)$ \\
\hline \multirow{3}{*}{3} & Parity \\
\hline & Nulliparous - $8(23 \%)$ \\
\hline & Multiparous - $27(77 \%)$ \\
\hline \multirow{3}{*}{4} & Performance status (ECOG) \\
\hline & $1-32(92 \%)$ \\
\hline & $2-3(8 \%)$ \\
\hline \multirow{4}{*}{5} & Co morbidities \\
\hline & Diabetes - $13(37 \%)$ \\
\hline & Hypertension-14 (40\%) \\
\hline & Metabolic syndrome- $6(17 \%)$ \\
\hline \multirow{5}{*}{6} & BMI \\
\hline & Normal - $13(37 \%)$ \\
\hline & Overweight $-16(46 \%)$ \\
\hline & Obese - $5(14 \%)$ \\
\hline & Morbidly obese - $1(3 \%)$ \\
\hline \multirow{4}{*}{7} & Presenting complaints \\
\hline & Menorrhagia - $7(20 \%)$ \\
\hline & Postmenopausal bleeding - $26(74 \%)$ \\
\hline & Others - $2(6 \%)$ \\
\hline \multirow{4}{*}{8} & Ultrasound findings \\
\hline & Normal - $6(17 \%)$ \\
\hline & Suspicious - $21(60 \%)$ \\
\hline & Highly suspicious - 8 (23\%) \\
\hline \multirow{3}{*}{9} & Type of uterine cancer \\
\hline & Type $1-31(89 \%)$ \\
\hline & Type $2-4(11 \%)$ \\
\hline \multirow{3}{*}{10} & Surgery performed \\
\hline & Laparoscopic - 5 (14\%) \\
\hline & Laparotomy - $30(86 \%)$ \\
\hline \multirow{4}{*}{11} & Lymphadenectomy \\
\hline & Not done - $5(14 \%)$ \\
\hline & Pelvic - $24(68 \%)$ \\
\hline & Pelvic and para-aortic - $6(17 \%)$ \\
\hline \multirow{4}{*}{12} & Omentectomy \\
\hline & Resected - $29(83 \%)$ \\
\hline & Sampling only - $3(8 \%)$ \\
\hline & Not sampled - $3(8 \%)$ \\
\hline \multirow{6}{*}{13} & Final FIGO stage (2009) \\
\hline & Stage $\quad 0-3(8 \%)$ \\
\hline & IA - $12(34 \%)$ \\
\hline & IB - $9(26 \%)$ \\
\hline & II - $6(17 \%)$ \\
\hline & III - $5(14 \%)$ \\
\hline
\end{tabular}


Table 4: Accuracy of MRI in comparison to final histology.

\begin{tabular}{|llll|}
\hline Accuracy & Myometrial invasion $(\mathrm{n}=35)$ & Nodes $(\mathrm{n}=32)$ & Cervical invasion $(\mathrm{n}=35)$ \\
\hline Sensitivity $(95 \% \mathrm{CI})$ & $0.70(0.46-0.88)$ & $0.67(0.09-0.99)$ & $0.43(0.10-0.82)$ \\
\hline Specificity $(95 \% \mathrm{CI})$ & $0.53(0.27-0.79)$ & $0.83(0.64-0.94)$ & $0.93(0.77-0.99)$ \\
\hline PPV $(95 \% \mathrm{CI})$ & $0.67(0.43-0.85)$ & $0.29(0.04-0.71)$ & $0.60(0.15-0.95)$ \\
\hline NPV $(95 \% \mathrm{CI})$ & $0.57(0.29-0.82)$ & $0.96(0.80-1.00)$ & $0.87(0.69-0.96)$ \\
\hline + LR & 1.50 & 3.94 & 6.14 \\
\hline - LR & 0.57 & 0.40 & 0.61 \\
\hline Overestimation & $7(20 \%)$ & $5(16 \%)$ & $2(6 \%)$ \\
\hline Underestimation & $6(17 \%)$ & $1(3 \%)$ & $4(11 \%)$ \\
\hline Accuracy & 0.63 & 0.81 & 0.83 \\
\hline
\end{tabular}

Table 5: Concordance of MRI and final pathology staging.

\begin{tabular}{|llll|}
\hline MIRI stage & Concordance with final pathology stage & Upstaged & Down staged \\
\hline 0 & 1 & 1 & 0 \\
\hline Ia & 6 & 0 & 1 \\
\hline Ib & 6 & 3 & 4 \\
\hline II & 3 & 1 & 1 \\
\hline IIIa & 0 & 1 & 2 \\
\hline IIIc & 1 & 0 & 4 \\
\hline
\end{tabular}

Table 6: Comparison of preoperative and postoperative risk grouping.

\begin{tabular}{|c|c|c|c|c|}
\hline Risk grouping & $\begin{array}{l}\text { Preoperative (histology, } \\
\text { MRI, Ca125) }\end{array}$ & $\begin{array}{l}\text { Postoperative } \\
\text { (histology, age) }\end{array}$ & Overestimation & Underestimation \\
\hline Low & $6(17 \%)$ & $15(43 \%)$ & $9(26 \%)$ & 0 \\
\hline Intermediate low & NA & $10(29 \%)$ & 0 & 0 \\
\hline Intermediate high & NA & $3(8 \%)$ & 0 & 0 \\
\hline High & $29(83 \%)$ & $7(20 \%)$ & 0 & 0 \\
\hline
\end{tabular}

\section{Preoperative risk grouping}

When MRI, Ca125 and histology were combined together, $29(83 \%)$ patients were deemed to be high risk to warrant a lymph node dissection. MRI alone contributed to high risk in $16(46 \%)$ patients. Histology alone contributed to high risk in $1(3 \%)$ patient. Ca-125 alone contributed to high risk in $1(3 \%)$ patient. MRI and Ca-125 together contributed to high risk in 3 pts $(8 \%)$. When histology was combined with MRI, 7 patients (20\%) were deemed to be high risk .1 (3\%) patient had all 3 risk factors to be grouped as high risk.

\section{Extent of surgery based on risk grouping}

All patients underwent hysterectomy and bilateral salpingo-oophorectomy as the least procedure. Fertility sparing surgery was not done in any patient. $32(91 \%)$ patients underwent pelvic lymphadenectomy and 5 (14.3\%) patients underwent both pelvic and para aortic lymphadenectomy. Omentectomy was done in 29 patients (83\%) and omental sampling was done in 3 patients. 3 patients had no resection or sampling of the omentum. Peritoneal cytology was negative in all patients and no omental metastases was detected in any patient. 4 patients $(11 \%)$ had a change of plan intraoperatively with significant looking nodes and underwent pelvic lymph node dissection although deemed to be low risk as per preoperative staging. The final histological examination of the lymph nodes was negative in these 4 patients. There was no major perioperative morbidity or mortality. 1 morbidly obese patient developed an umbilical port site hernia 6 months after the laparoscopic staging procedure and underwent a mesh repair.

\section{Effect of risk groupings}

Postoperatively $20(57 \%)$ patients were grouped as high risk and the rest as low risk. Histological features alone contributed to high risk in 10 patients $(28 \%)$ and nodal invasion contributed to high risk in $3(9 \%)$ patients. Age as a risk factor contributed to Intermediate high risk in 7 patients $(20 \%)$ with low risk histology. When compared to the preoperative staging, 9 patients were down staged and none were upstaged giving a $40 \%$ sensitivity, $100 \%$ specificity, $100 \%$ PPV and 69\% NPV (Table 6). 
The 20 high risk patients were advised adjuvant treatment. 2 patients had adjuvant chemoradiation. 4 patients had adjuvant EBRT and brachytherapy. 2 patients received EBRT alone. 1 patient had brachytherapy alone. $4(11 \%)$ patients underwent chemotherapy alone. 1 patient did not complete the prescribed course of RT. 6 patients refused to undergo the prescribed adjuvant brachytherapy.

The median follow-up time was 20 months with range 658 months. There were no vault recurrences. There were 5 deaths $(14 \%)$ with 3 disease related mortality and 2 non disease related mortality. All the mortality seen was in the high-risk group. 1 patient in the low risk group was detected with metachronous breast cancer and 1 patient in the intermediate high-risk group was detected with metachronous medullary carcinoma thyroid. 2 patients are alive with disease at the conclusion of this study (liver and lung metastases) and 1 patient was lost to follow up after adjuvant RT.

\section{DISCUSSION}

The patient cohort in this study had a median age of 56 years with the youngest patient aged 42 years. Similar studies of endometrial cancer in Indian women report a median age of 54 years and 59 years respectively from two studies reported from western and southern India., A study of early stage endometrial carcinomas with similar native population from northern Kerala has reported a median age of 59 years. ${ }^{7}$ This is a decade earlier than in US where median age of diagnosis is 63 years. $^{8}$

Carcinosarcomas have been recently re grouped under endometrial cancers in the recent re-classification of endometrial cancers. ${ }^{9}$ Carcinosarcoma is known to have an aggressive behavior despite early stage at presentation and adjuvant treatment has been mostly futile in most studies. ${ }^{10}$ Anupama at al describe a retrospective series with "less favourable outcomes". ${ }^{11}$ The four patients with carcinosarcoma initially included in this study had a high morbidity and mortality and were excluded from the final analysis as the authors feel that this histology should be treated as a separate entity. ${ }^{9}$

Preoperative histology is the single most important determinator of preoperative risk. Well differentiated or low grade endometrioid cancers are known to behave well prognostically as compared to aggressive histologies while carcinosarcomas are known to have poor outcomes despite early stage at presentation. ${ }^{12}$ Low risk histology needs only an extra fascial hysterectomy whereas high risk histologies warrant a comprehensive staging procedure despite apparent early stage disease on MRI and normal $\mathrm{Ca} 125$ levels.

Pap smears have not been used in the screening of endometrial cancer. When atypical glandular cytology is detected, up to $38 \%$ may harbour malignancy in the cervix or endometrium. ${ }^{13}$ In this study pap smear was not a requirement for inclusion as patients had already been diagnosed with endometrial curettage. Reviewing the data, we could see that only 9 patients $(26 \%)$ had undergone a pap smear prior to the endometrial curettage and 3 of these smears $(33 \%)$ showed atypical glandular cells. 1 patient $(11 \%)$ had a pap smear detected endometrial cancer. The literature says Pap smear has $71 \%$ chance of picking up malignancy especially in the post-menopausal age group. ${ }^{14}$

To obtain preoperative histology conventional endometrial curettage has been the practice in our institution and hence all patients in this study had a formal uterine curettage than an office endometrial biopsy. Many centres now practice office endometrial biopsy and it is said to be a more cost-effective strategy to evaluate postmenopausal bleeding. Accuracy of office biopsy is lower in premenopausal women and they need a formal uterine curettage. ${ }^{15}$

The difficulty in practice is the accuracy of preoperative histology which can be quite varied especially outside major institutions. Fear of underestimation of risk in preoperative histology leads to blanket surgical staging procedures which is a dent on resources both for patients and institutions. The accuracy of preoperative histology by formal endometrial curetting was $73 \%$ in this study with more overestimation than underestimation. Accuracy rates are said to be better with hysteroscopy assisted endometrial sampling. ${ }^{16}$

MRI has become a key investigation modality in the management of endometrial cancers. Diffusion weighted imaging and contrast enhanced MR imaging adds information to the standard morphological imaging and helps to improve staging accuracy. ${ }^{17}$ The guidelines do not mandate preoperative MRI, but it is often used to obtain more accurate information especially with regards to myometrial and cervical invasion. In apparent stage I endometrial cancer, MRI may be useful to complete information regarding myometrial invasion. ${ }^{18}$ The EsmoEstro guidelines encourages the use of MRI in institutions where surgery can be tailored according to the risk groups. ${ }^{2}$ Several studies have looked into the accuracy of MRI in the staging of endometrial cancers. From the literature, the overall staging accuracy of MRI is $83-92 \%$ with wide variation in the rates. ${ }^{19}$ This accuracy is calculated from the three commonly studied parameters of myometrial invasion, cervical invasion and nodal metastasis. $^{20}$

The sensitivity of MRI in detecting myometrial invasion has been in the range of $56-92 \% .^{19,20}$ In this study, sensitivity, specificity of myometrial invasion is lower than quoted in literature. The accuracy for cervical invasion and nodal metastases is on par with other similar studies. Specificity of cervical invasion and negative predictive value of nodal invasion was the highest. Some studies have shown that the detection of cervical invasion 
has been reported to have low accuracy. ${ }^{21}$ In this study, cervical invasion had a specificity of 93\%. Negative predictive value of cervical invasion and nodal invasion was high in this study but accuracy for myometrial invasion was low with higher sensitivity and lower specificity than quoted in literature. ${ }^{22}$ While many studies in the literature quote high accuracy rates for myometrial invasion, Anderson et al and Rockall et al have reported similar rates of accuracy for these parameters as in our study with specificity and negative predictive value for cervical invasion and nodal invasion being in the range of $75-98 \%$. $^{23,24}$

\section{Effect of combining histology, Ca 125 and MRI for preoperative risk grouping}

Histology and MRI without Ca 125 for preoperative stratification by Luomaranta et al concluded that this strategy was only moderately sensitive and specific and there was risk of missing significant number of high-risk women. ${ }^{25} \mathrm{Ca} 125$ has been found to be an independent marker for risk stratification especially in low grade endometrial cancer. ${ }^{26,27}$

Histology, MRI stage, Ca-125 have been used to stratify risk preoperatively with authors reporting feasibility in retrospective studies. ${ }^{28,29}$ Tumor index and more recently Immunohistochemistry markers have been added to the preoperative triaging methods. ${ }^{30}$ The GOG-99, the Mayomodified, and the ESMO-modified criteria (with combinations of these parameters ) have been compared for preoperative risk stratification and the ESMO model was seen to be the most accurate. ${ }^{31}$ However, the ESMO model requires LVSI as a determinator of risk. This information is not available in routine preoperative histology sampling and places additional cost and resources to obtain it outside tertiary cancer centres.

In this study, there was $26 \%$ overestimation of risk but no underestimation by the preoperative triaging method. The PPV and specificity was high with low sensitivity and moderate NPV. There is emphasis on freedom to change the extent of surgical staging as necessary based on intraoperative findings especially with respect to the pelvic and para aortic nodal staging procedure. Luomaranta et al have studied the feasibility of omitting or completing para aortic nodal dissection based on gross findings of the pelvic nodes at surgery. In 854 women studied, there was $98.4 \%$ negative predictive value. ${ }^{32}$ in this study, 4 low risk patients had a complete node dissection after finding gross nodes at surgery. But final pathology was negative for metastases in all of them.

For the rare miliary omental or peritoneal metastases that may not be detected by the preoperative imaging, intraoperative exploration does provide the opportunity to complete the staging procedure. No patient had omental metastases in this study. Rather, two patients with carcinosarcoma who had been excluded from the analysis had positive omental metastases. Peritoneal cytology was negative in all patients in this study, but positive in the excluded carcinosarcoma patients. Peritoneal cytology is no longer mandatory according to the guidelines and omental sampling or resection is recommended for high risk histologies only. ${ }^{2}$ The authors suggest that omental sampling or resection can be safely omitted in endometrial cancers if grossly normal at surgery.

\section{Oncological outcomes}

No isolated local or vault recurrence was seen in this study. It is possible that we have not reached the time for vault recurrence as the median time to recurrence is 32 months and the median follow up here is only 20 months. ${ }^{33}$ Vault recurrence is primarily based on initial risk grouping and patients naive to radiotherapy in first line treatment responded better to salvage treatment. ${ }^{34}$ In the recent update of follow up guidelines for endometrial cancer ESTRO-ESMO guidelines dropped the mandatory vault smears at each visit as these were not cost effective. $^{2}$

All the mortality in this study was in high risk group. The morbidity in the low risk group was seen as metachronous cancers. The lack of adjuvant radiotherapy in patients deemed intermediate high risk due to age alone did not seem to have a detrimental effect in this study cohort.

\section{CONCLUSION}

The preoperative risk stratification using preoperative histology, Ca 125 and MRI and tailoring surgical staging accordingly does not seem to have had a negative effect on oncological outcomes in this study. None of the patients deemed as low risk preoperatively had been upstaged to high risk postoperatively, thereby not affecting adjuvant treatment patterns. While the small sample size is a limitation to draw any conclusions, it reflects current practice and future directions. Larger studies will help to validate these methods and be incorporated into formal guidelines.

\section{Funding: No funding sources}

Conflict of interest: None declared

Ethical approval: The study was approved by the Institutional Ethics Committee

\section{REFERENCES}

1. India state-level disease burden initiative cancer collaborators. The burden of cancers and their variations across the states of India: The Global Burden of Disease Study 1990-2016, The Lancet Oncol. 2018;19:10:1289-306.

2. Colombo N, Creutzberg C, Amant F. ESMO-ESGOESTRO consensus conference on endometrial cancer: Diagnosis, treatment and follow-up. Radiother Oncol. 2015;117:559-81. 
3. Tsikouras P, Koukouli Z, Bothou A, Manav B, Iatrakis G, Zervoudis $S$, et al.Preoperative assessment in endometrial cancer. Is triage for lymphadenectomy possible? J BUON. 2017;22(1):34-43.

4. Reijnen C, Visser NC, Kasius JC. Improved preoperative risk stratification with CA-125 in lowgrade endometrial cancer: a multicenter prospective cohort study. J Gynecol Oncol. 2019;30(5):e70.

5. Mahantshetty U, Aggarwal A, Ganesh B, Saoba S, Mulla S, Engineer R, et al. Clinical outcome of earlystage endometrioid adenocarcinoma: A tertiary cancer center experience. Int J Gynecol Cancer. 2013;23:1446-52.

6. Balasubramaniam G, Sushama S, Rasika B, Mahantshetty U. Hospital-based study of endometrial cancer survival in Mumbai, India. Asian Pac J Cancer Prev. 2013;14(2):977-80.

7. Dessai S, Adrash D, Geetha M, Arvind S, Bipin J, Nayanar S, et al. Pattern of care in operable endometrial cancer treated at a rural-based tertiary care cancer center. Indian J Cancer. 2016;53:416-9.

8. Lee NK, Cheung MK, Shin JY. Prognostic factors for uterine cancer in reproductive-aged women. Obstet Gynecol. 2007;109:655-62.

9. Cantrell LA, Blank SV, Duska LR, Uterine carcinosarcoma: a review of the literature. Gynecol. Oncol. 2015;137:581-8.

10. Mendivil A, Schuler KM, Gehrig PA. Nonendometrioid adenocarcinoma of the uterine corpus: a review of selected histological subtypes. Cancer Control. 2009;16(1):46-52.

11. Anupama R, Kuriakose S, Vijaykumar DK. Carcinosarcoma of the uterus-a single institution retrospective analysis of the management and outcome and a brief review of literature. Indian $\mathbf{J}$ Surg Oncol. 2013;4(3):222-8.

12. Pessini SA, Zettler CG, Wender MC, Pellanda LC, Silveira GP. Survival and prognostic factors of patients treated for Stage I to Stage III endometrial carcinoma in a reference cancer center in Southern Brazil. Eur J Gynaecol Oncol. 2007;28:48-50.

13. DeSimone CP, Day ME, Tovar MM, Dietrich CS 3rd, Eastham ML, Modesitt SC. Rate of pathology from atypical glandular cell Pap tests classified by the Bethesda 2001 nomenclature. Obstet Gynecol. 2006;107(6):1285-91.

14. Nadaf A, Rani H, Rao R, Shastri D. Pap smears in endometrial adenocarcinoma: does it have a role? Asian Pac J Cancer Prev. 2017;18(4):1145-50.

15. Dijkhuizen FP, Mol BW, Brölmann HA, Heintz AP. The accuracy of endometrial sampling in the diagnosis of patients with endometrial carcinoma and hyperplasia: a meta-analysis. Cancer. 2000;89(8):1765-72.

16. Al-Talib A, Nezhat F, Tulandi T. The role of hysteroscopy in the diagnosis and management of endometrial cancer. Gynecol Surg. 2010;7:211-6.

17. Beddy P, O’Neil AC, Yamamoto AK, Addley HC, Reinhold C, Sala E. FIGO Staging system for endometrial cancer: added benefits of MR Imaging. Radio Graphics. 2012;32:241-54.

18. Epstein E, Blomqvist L. Imaging in endometrial cancer. Best Pract Res Clin Obstet Gynaecol. 2014;28:721-39.

19. Faria SC, Sagebiel T, Balachandran A, Devine C, Lal $\mathrm{C}$, Bhosale PR. Imaging in endometrial carcinoma. Indian J Radiol Imaging. 2015;25:137-47.

20. Shrivastava S, Barmon D, Kataki AC, Deka P, Sharma JD, Choudhary BK, et al. Magnetic resonance imaging in pre-operative staging of endometrial cancer. Indian J Cancer. 2016;53:181-5.

21. Ortashi O, Jain S, Emannuel O, Henry R, Wood A, Evans J. Evaluation of the sensitivity, specificity, positive and negative predictive values of preoperative magnetic resonance imaging for staging endometrial cancer: a prospective study of 100 cases at the Dorset Cancer Centre. Eur J Obstet Gynecol Reprod Biol. 2008;137(2):232-5.

22. Wu WJ, Yu MS, Su HY, Lin KS, Lu KL, Hwang KS. The accuracy of magnetic resonance imaging for preoperative deep myometrium assessment in endometrial cancer. Taiwan J Obstet Gynecol. 2013;52(2):210-4.

23. Anderson J, Shanbhag S, Cruikshank D. Use and accuracy of magnetic resonance imaging (MRI) staging of network treated endometrial cancer (EC): An audit and comparison to best evidence Sri Lanka J Obstet Gynaecol. 2009;31:104-7.

24. Rockall AG, Meroni R, Sohaib SA, Reynolds K, Alexander-Sefre F, Shepherd JH, et al. Evaluation of endometrial carcinoma on magnetic resonance imaging. Int J Gynecol Cancer. 2007;17:188-96.

25. Luomaranta A, Bützow R, Pauna AR, Leminen A, Loukovaara M. Combined use of endometrial sample and magnetic resonance imaging in the preoperative risk-stratification of endometrial carcinomas. Acta Obstet Gynecol Scand. 2015;94(1):95-101.

26. Todo Y, Sakuragi N, Nishida R, Yamada T, Ebina Y, Yamamoto R, et al. Combined use of magnetic resonance imaging, CA 125 assay, histologic type, and histologic grade in the prediction of lymph node metastasis in endometrial carcinoma. Am J Obstet Gynecol. 2003;188(5):1265-72.

27. Mitamura T, Watari H, Todo Y, Kato T, Konno Y, Hosaka $\mathrm{M}$, et al. Lymphadenectomy can be omitted for low-risk endometrial cancer based on preoperative assessments. J Gynecol Oncol. 2014:PMC4195300

28. Lavoué V, Body N, Kerdaniel OD, Foucher F, Henno S, Cauchois A. Are preoperative histology and MRI useful for classification of endometrial cancer risk?, BMC Cancer. 201616:498:PMCID: PMC4950781.

29. Todo Y, Sakuragi N, Nishida R, Yamada T, Ebina Y, Yamamoto R, et al. Combined use of magnetic resonance imaging, CA 125 assay, histologic type, and histologic grade in the prediction of lymph node metastasis in endometrial carcinoma. Am J Obstet Gynecol. 2003;188(5):1265-72. 
30. Vit W, Marketa B, Jitka H, Petra O, Petra V, Lubos $\mathrm{M}$ et al. A novel approach to preoperative risk stratification in endometrial cancer: the added value of immunohistochemical markers, 2019. Available at: https://doi.org/10.3389/fonc.2019.00265.

31. Korkmaz V, Meydanli MM, Yalçın I. Comparison of three different risk-stratification models for predicting lymph node involvement in endometrioid endometrial cancer clinically confined to the uterus. J Gynecol Oncol. 2017;28(6):e78.

32. Luomaranta A, Lohi J, Bützow R, Leminen A, Loukovaara M. Prediction of para-aortic spread by gross pelvic lymph node findings in patients with endometrial carcinoma. Int $\mathbf{J}$ Gynecol Cancer. 2014;24(4):697-702.

33. Robbins JR, Yechieli R, Laser B, Mahan M, Rasool $\mathrm{N}$, Elshaikh MA. Is time to recurrence after hysterectomy predictive of survival in patients with early stage endometrial carcinoma? Gynecol Oncol. 2012;127:38-42.

34. Sorbe B. Juresta C, Ahlin C. Natural history of recurrences in endometrial carcinoma. Oncol Lett. 2014:PMID:PMC415626.

Cite this article as: Mathews J, Abraham S. Early oncological outcomes and accuracy of risk stratification and tailoring surgical staging based on preoperative histology, Ca125 and MRI in endometrial cancer: a prospective cohort study. Int J Reprod Contracept Obstet Gynecol 2019;8:4324-31. 